\title{
Interstellar Chemistry: A Strategy for Detecting Polycyclic Aromatic Hydrocarbons in Space
}

\author{
F. J. Lovas, ${ }^{1}$ Robert J. McMahon, ${ }^{2 *}$ Jens-Uwe Grabow,${ }^{3}$ Melanie Schnell, ${ }^{3}$ \\ James Mack, ${ }^{\#, \&}$ Lawrence T. Scott, ${ }^{\#}$ Robert L. Kuczkowski ${ }^{\perp}$ \\ Optical Technology Division, National Institute of Standards and Technology, Gaithersburg, \\ MD 20899; Department of Chemistry, University of Wisconsin, Madison, WI 53706; Institut für \\ Physikalische Chemie, Universität Hannover, D-30167 Hannover, Germany; Department of \\ Chemistry, Merkert Chemistry Center, Boston College, Chestnut Hill, MA 02467; Department of \\ Chemistry, University of Michigan, Ann Arbor, MI 48109
}

\section{Supporting Information Contents}

Discussion of Centrifugal Distortion Constants

p. 2

Discussion of Corannulene Bowl Inversion Process

p. 4

References

p. 4

Table S1. Calculated and Measured Rotational Transitions of Corannulene $\left(\mathrm{C}_{20} \mathrm{H}_{10}\right)$ to $J=154(158 \mathrm{GHz})$.

p. 5-8

\footnotetext{
${ }^{1}$ National Institute of Standards and Technology.

${ }^{2}$ University of Wisconsin.

${ }^{3}$ Universität Hannover.

" Boston College.

${ }^{\perp}$ University of Michigan.

${ }^{\&}$ Present Address: Department of Chemistry, University of Cincinnati, Cincinnati, OH 45221, USA.
} 


\section{Centrifugal Distortion Constants}

The statistical weights for the $K$ states of corannulene were determined using the analysis of Weber. ${ }^{1}$ Corannulene $\left(C_{5 \mathrm{v}}\right)$ has atoms of type 5 in Table I of Weber. ${ }^{1}$ Using Eq. (3) with $h=$ 10 (order of the group), Eqs. (4), (8), and (9) afford values for $\chi_{S}(R)$, and group theory analysis affords values for $\chi_{G}{ }^{\alpha}(R){ }^{2}$ The resulting $m_{\alpha}$ values are: $m\left(\mathrm{~A}_{1}\right)=120 ; m\left(\mathrm{~A}_{2}\right)=88 ; m\left(\mathrm{E}_{1}\right)=m\left(\mathrm{E}_{2}\right)$ $=204$. Then from the first column of Table $\mathrm{V}^{1}$ for the $\mathrm{A}_{1}$ ro-vibronic ground state:

$$
\begin{array}{ll}
\boldsymbol{K} & \text { weight } \\
0 & m\left(\mathrm{~A}_{1}\right)+m\left(\mathrm{~A}_{2}\right)=208 \\
5 \mathrm{p}=5,10, \ldots & 2\left[m\left(\mathrm{~A}_{1}\right)+m\left(\mathrm{~A}_{2}\right)\right]=416 \\
5 \mathrm{p} \pm 1=1,4,6,9,11, \ldots & 2 m\left(\mathrm{E}_{1}\right)=408 \\
5 \mathrm{p} \pm 2=2,3,7,8,12, \ldots & 2 m\left(\mathrm{E}_{2}\right)=408
\end{array}
$$

Thus, the weighting of the $K=0$ state is about half of all the other $K$ states, and the relative magnitudes of the transition matrix elements follow the formula $1-\left[K^{2} /(J+1)^{2}\right]$.

The analysis reveals that corannulene displays three symmetry species $\left(A_{1}+A_{2}\right), E_{1}$, and $\mathrm{E}_{2}$, and cooling in the beam expansion might populate only the states available with energies up to the order of kT. In the case that $D_{J K}$ is extremely small, the analysis is straightforward and the determined constants are $B$ and $D_{J}$. In the case that $D_{J K}$ is not small, the proper determination of $D_{J}$ and $D_{J K}$ becomes problematic. If $D_{J K}$ is not small, the value determined for $D_{J}$ is just an effective value, since the shift attributed to $D_{J}$ might be caused by the population of higher $K$ states (and depopulation of lower $K$ states) as they become available with higher $J$. In the extreme case, only the three highest $K$ states are populated after the transfer during the supersonic expansion. (If $D_{J K}$ is sufficiently small, however, one would still see only one line in any $J \rightarrow J+1$ transition). If the population transfer is not complete, more $K$ states would be 
populated, but the "mean" $K$-value would not be zero. Most likely, $K$ will be a function of $J$, e.g. $K=J-\mathrm{X}$ with $J^{2}>(J-X)^{2}>\frac{k T}{C-B}+J^{2}>0$, since states with a relative energy beyond the order of kT will essentially not be populated (e.g. for $J$ levels with $J^{2}>\frac{k T}{B-C}$ the population of the $K=0$ state will decrease significantly; i.e. $J>14$ assuming a beam temperature of $2.5 \mathrm{~K}$ ). Irrespective of whether or not population transfer is complete, a simple interpretation of this situation in terms of $D_{J}$ and $D_{J K}$ is not possible. In such a case, the value of $D_{J}$ is just as speculative as $D_{J K}$, since it is not easily possible to distinguish in a fit between $D_{J} \times J^{2}(J+1)^{2}$ and $D_{J K} \times J(J+1) K^{2}$ approximately equal to $D_{J K} \times J(J+1)(J-\mathrm{X})^{2}$.

The Stark effect measurements, however, permit a definite statement on $D_{J}$. From the Stark measurements, the $K=0$ line position was extrapolated to zero-field conditions. Indeed, the extrapolated line coincides with the measured zero-field line position (Fig 4). This indicates the absence of a line shift arising from a mixture of different overlapping $K$-states, which confirms the small magnitude of $D_{J K}$. We therefore conclude that the line position for higher $J$ transitions is not affected by a mix of overlapping $K$-states.

From the line width at half maximum intensity for the highest frequency transition observed, a value of $0.0023 \mathrm{kHz}$ for $D_{J K}$ is determined as an upper limit to the splitting from $K=$ 0 to $K=18$. This limit is half of the value of $D_{J}$. The upper limit for $D_{J K}$ should probably be increased somewhat, since the highest $K$ values are likely too weak to observe in the laboratory spectrum. In the end, extrapolation of the transition frequencies to the mm range (e.g. 75-100 $\mathrm{GHz}$ ), as well as conclusions from the mm survey data, should be considered with due caution. 


\section{Corannulene Bowl Inversion Process}

Inversion along the c-axis (dipolar axis) would provide two levels for each $\mathrm{J}$ state.

Transitions from $\mathrm{J}^{\prime \prime}$ to $\mathrm{J}^{\prime}$ would go from the bottom level in the lower state ( $\mathrm{J}$ ") to the upper level in the upper state $\left(\mathrm{J}^{\prime}\right)$, and vice versa for the other two levels. Thus, the transitions go across the inversion splitting and the observed frequency splitting (if it happened) would be twice the inversion frequency. The narrowest linewidth measured in the current study is $9 \mathrm{kHz}$, which places an upper limit to the inversion frequency of $5 \mathrm{kHz}$ at the temperature of the measurement $(<2 \mathrm{~K})$. (The rotational temperature in the supersonic expansion is estimated to be 1-2 K).

From comparison with other systems, the low inversion frequency $(<5 \mathrm{kHz})$ seems reasonable for a large reduced mass tunneling through a barrier of $10.2 \mathrm{kcal} / \mathrm{mol}$ (measured for an analog of corannulene by dynamic NMR spectroscopy. ${ }^{3}$ ) Extrapolation of the measured rate to temperatures below $100 \mathrm{~K}$ predicts a vanishingly low rate of inversion via a classical, thermallyactivated process. The absence of inversion splittings in the laboratory rotational spectra has no implication regarding astronomical observations, particularly for hot sources, which have broad lines. Dark clouds have narrower lines, which are, nevertheless, comparable to the $9 \mathrm{kHz}$ width observed in Hannover.

\section{References}

(1) Weber, A. J. Chem. Phys. 1982, 76, 3694-3698.

(2) Wilson, E. B., Jr.; Decius, J. C.; Cross, P. C. Molecular Vibrations; Dover: New York, 1955.

(3) Scott, L. T.; Hashemi, M. M.; Bratcher, M. S. J. Am. Chem. Soc. 1992, 114, 1920-1921. 
Table S1. Calculated and Measured Rotational Transitions of Corannulene $\left(\mathrm{C}_{20} \mathrm{H}_{10}\right)$.

\begin{tabular}{|c|c|c|c|c|c|}
\hline J' - J" & $\begin{array}{c}\text { Calculated } \\
\text { Frequency } \\
\text { [MHz] }\end{array}$ & $\begin{array}{l}\text { uncertainty } \\
\qquad(2 \sigma)\end{array}$ & $\begin{array}{c}\text { Measured } \\
\text { Frequency } \\
{[\mathrm{MHz}]}\end{array}$ & $\begin{array}{l}\text { uncertainty } \\
\qquad(1 \sigma)\end{array}$ & $\begin{array}{c}\text { Energy of } \\
\text { Lower State } \\
{\left[\mathrm{cm}^{-1}\right]}\end{array}$ \\
\hline $1-0$ & 1019.6854 & 1) & & & 0.000 \\
\hline $2-1$ & 2039.3706 & 1) & & & 0.034 \\
\hline $3-2$ & 3059.0556 & 2) & & & 0.102 \\
\hline $4-3$ & 4078.7404 & 2) & & & 0.204 \\
\hline $5-4$ & 5098.4247 & 2) & 5098.4246 & ( 5$)$ & 0.340 \\
\hline $6-5$ & 6118.1084 & 3) & 6118.1081 & ( 5 ) & 0.510 \\
\hline $7-6$ & 7137.7916 & 3) & 7137.7916 & ( 5 ) & 0.714 \\
\hline $8-7$ & 8157.4740 & 3) & 8157.4739 & ( 5 ) & 0.952 \\
\hline $9-8$ & 9177.1556 & 3) & 9177.156 & ( 2 ) & 1.224 \\
\hline $10-9$ & 10196.8363 & 4) & 10196.838 & ( 2) & 1.531 \\
\hline $11-10$ & 11216.5159 & 4) & 11216.517 & ( 2) & 1.871 \\
\hline $12-11$ & 12236.1943 & 4) & 12236.197 & ( 2$)$ & 2.245 \\
\hline $13-12$ & 13255.8715 & 5) & 13255.872 & ( 2) & 2.653 \\
\hline $14-13$ & 14275.5473 & 6) & 14275.549 & ( 2) & 3.095 \\
\hline $15-14$ & 15295.2217 & 7) & 15295.222 & ( 2) & 3.571 \\
\hline $16-15$ & 16314.8945 & 8) & 16314.894 & ( 2) & 4.082 \\
\hline $17-16$ & 17334.5657 & 10) & 17334.564 & ( 2) & 4.626 \\
\hline $18-17$ & 18354.2350 & 12) & 18354.234 & ( 2) & 5.204 \\
\hline $19-18$ & 19373.9025 & 15) & 19373.903 & ( 2) & 5.816 \\
\hline $20-19$ & 20393.5680 & 18) & & & 6.462 \\
\hline $21-20$ & 21413.2314 & 21) & & & 7.143 \\
\hline $22-21$ & 22432.8926 & 25) & & & 7.857 \\
\hline $23-22$ & 23452.5515 & 29) & & & 8.605 \\
\hline $24-23$ & 24472.2080 & 33) & & & 9.388 \\
\hline $25-24$ & 25491.8619 & 39) & & & 10.204 \\
\hline $26-25$ & 26511.5133 & 44) & & & 11.054 \\
\hline $27-26$ & 27531.1620 & 50) & & & 11.939 \\
\hline $28-27$ & 28550.8078 & 57) & & & 12.857 \\
\hline $29-28$ & 29570.4507 & 64) & & & 13.809 \\
\hline $30-29$ & 30590.0906 & 71) & & & 14.796 \\
\hline $31-30$ & 31609.7273 & 79) & & & 15.816 \\
\hline $32-31$ & 32629.3608 & 88) & & & 16.870 \\
\hline $33-32$ & 33648.9910 & 97) & & & 17.959 \\
\hline $34-33$ & 34668.6177 & ( 107) & & & 19.081 \\
\hline $35-34$ & 35688.2408 & ( 118) & & & 20.238 \\
\hline $36-35$ & 36707.8603 & ( 129) & & & 21.428 \\
\hline $37-36$ & 37727.4760 & ( 141) & & & 22.652 \\
\hline $38-37$ & 38747.0879 & ( 153) & & & 23.911 \\
\hline $39-38$ & 39766.6957 & ( 166) & & & 25.203 \\
\hline $40-39$ & 40786.2995 & ( 180$)$ & & & 26.530 \\
\hline
\end{tabular}




\begin{tabular}{|c|c|c|}
\hline $41-40$ & 41805.8992 & 195) \\
\hline $42-41$ & 42825.4945 & 211) \\
\hline $43-42$ & 43845.0854 & 227) \\
\hline $44-43$ & 44864.6719 & 244) \\
\hline $45-44$ & 45884.2537 & 262) \\
\hline $46-45$ & 46903.8309 & 280) \\
\hline $47-46$ & 47923.4032 & $300)$ \\
\hline $48-47$ & 48942.9706 & 320) \\
\hline $49-48$ & 49962.5330 & 341) \\
\hline $50-49$ & 50982.0903 & 363) \\
\hline $51-50$ & 52001.6424 & $387)$ \\
\hline $52-51$ & 53021.1891 & 411) \\
\hline $53-52$ & 54040.7304 & 436) \\
\hline $54-53$ & 55060.2661 & 461) \\
\hline $55-54$ & 56079.7962 & 488) \\
\hline $56-55$ & 57099.3205 & 516) \\
\hline $57-56$ & 58118.8390 & 545) \\
\hline $58-57$ & 59138.3516 & 575) \\
\hline $59-58$ & 60157.8580 & 607) \\
\hline $60-59$ & 61177.3583 & 639) \\
\hline $61-60$ & 62196.8524 & 672) \\
\hline $62-61$ & 63216.3400 & 706) \\
\hline $63-62$ & 64235.8212 & 742) \\
\hline $64-63$ & 65255.2957 & 779) \\
\hline $65-64$ & 66274.7636 & 817) \\
\hline $66-65$ & 67294.2247 & 856) \\
\hline $67-66$ & 68313.6789 & 896) \\
\hline $68-67$ & 69333.1261 & 938) \\
\hline $69-68$ & 70352.5662 & 981) \\
\hline $70-69$ & 71371.9991 & 1025) \\
\hline $71-70$ & 72391.4246 & 1070) \\
\hline $72-71$ & 73410.8427 & 1117) \\
\hline $73-72$ & 74430.2533 & ( 1165) \\
\hline $74-73$ & 75449.6563 & ( 1214) \\
\hline $75-74$ & 76469.0515 & (1265) \\
\hline $76-75$ & 77488.4389 & 1317) \\
\hline $77-76$ & 78507.8183 & 1370) \\
\hline $78-77$ & 79527.1897 & ( 1425) \\
\hline $79-78$ & 80546.5530 & ( 1482) \\
\hline $80-79$ & 81565.9079 & ( 1540) \\
\hline $81-80$ & 82585.2545 & 1599) \\
\hline $82-81$ & 83604.5927 & 1660) \\
\hline $83-82$ & 84623.9223 & ( 1722) \\
\hline $84-83$ & 85643.2432 & 1786) \\
\hline $85-84$ & 86662.5553 & 1851) \\
\hline $86-85$ & 87681.8585 & 1918) \\
\hline $87-86$ & 88701.1527 & 1987) \\
\hline $88-87$ & 89720.4379 & ( 2057) \\
\hline $89-88$ & 90739.7138 & 2129) \\
\hline $90-89$ & 91758.9804 & ( 2202) \\
\hline
\end{tabular}

27.890

29.285

30.713

32.176

33.672

35.203

36.767

38.366

39.999

41.665

43.366

45.100

46.869

48.671

50.508

52.379

54.283

56.222

58.195

60.201

62.242

64.317

66.425

68.568

70.745

72.955

75.200

77.479

79.791

82.138

84.519

86.934

89.382

91.865

94.382

96.932

99.517

102.136

104.789

107.475

110.196

112.951

115.740

118.562

121.419

124.310

127.235

130.193

133.186

136.213 


\begin{tabular}{|c|c|c|}
\hline $91-90$ & 92778.2376 & ( 2277) \\
\hline $92-91$ & 93797.4854 & 2354) \\
\hline $93-92$ & 94816.7234 & 2432) \\
\hline $94-93$ & 95835.9518 & 2512) \\
\hline $95-94$ & 96855.1703 & 2594) \\
\hline $96-95$ & 97874.3790 & 2678) \\
\hline $97-96$ & 98893.5775 & 2763) \\
\hline $98-97$ & 99912.7660 & 2850) \\
\hline $99-98$ & 100931.9441 & 2939) \\
\hline $100-99$ & 101951.1120 & 3030) \\
\hline $101-100$ & 102970.2693 & 3123) \\
\hline $102-101$ & 103989.4162 & 3217) \\
\hline $103-102$ & 105008.5523 & 3314) \\
\hline $104-103$ & 106027.6777 & 3412) \\
\hline $105-104$ & 107046.7922 & $3512)$ \\
\hline $106-105$ & 108065.8957 & 3614) \\
\hline $107-106$ & 109084.9882 & 3719) \\
\hline $108-107$ & 110104.0695 & 3825) \\
\hline $109-108$ & 111123.1394 & 3933) \\
\hline $110-109$ & 112142.1980 & 4043) \\
\hline $111-110$ & 113161.2451 & 4155) \\
\hline $112-111$ & 114180.2806 & 4269) \\
\hline $113-112$ & 115199.3043 & 4385) \\
\hline $114-113$ & 116218.3163 & 4504) \\
\hline $115-114$ & 117237.3163 & 4624) \\
\hline $116-115$ & 118256.3043 & 4746) \\
\hline $117-116$ & 119275.2802 & 4871) \\
\hline $118-117$ & 120294.2438 & 4998) \\
\hline $119-118$ & 121313.1952 & 5127) \\
\hline $120-119$ & 122332.1340 & 5258) \\
\hline $121-120$ & 123351.0604 & 5392) \\
\hline $122-121$ & 124369.9740 & $5527)$ \\
\hline $123-122$ & 125388.8749 & 5665) \\
\hline $124-123$ & 126407.7630 & 5805) \\
\hline $125-124$ & 127426.6381 & 5948) \\
\hline $126-125$ & 128445.5001 & 6092) \\
\hline $127-126$ & 129464.3490 & 6239) \\
\hline $128-127$ & 130483.1846 & 6389) \\
\hline $129-128$ & 131502.0067 & 6541) \\
\hline $130-129$ & 132520.8155 & 6695) \\
\hline $131-130$ & 133539.6106 & 6851) \\
\hline $132-131$ & 134558.3920 & 7010) \\
\hline $133-132$ & 135577.1596 & 7171) \\
\hline $134-133$ & 136595.9133 & 7335) \\
\hline $135-134$ & 137614.6530 & 7502) \\
\hline $136-135$ & 138633.3786 & 7670) \\
\hline $137-136$ & 139652.0900 & 7842) \\
\hline $138-137$ & 140670.7870 & 8016) \\
\hline $139-138$ & 141689.4696 & 8192) \\
\hline $140-139$ & 142708.1377 & (8371) \\
\hline
\end{tabular}

139.274

142.368

145.497

148.660

151.857

155.087

158.352

161.651

164.984

168.350

171.751

175.186

178.654

182.157

185.694

189.265

192.869

196.508

200.181

203.887

207.628

211.403

215.211

219.054

222.931

226.841

230.786

234.764

238.777

242.823

246.904

251.019

255.167

259.350

263.566

267.817

272.101

276.420

280.772

285.158

289.579

294.033

298.522

303.044

307.600

312.191

316.815

321.473

326.166

330.892 


\begin{tabular}{llll}
$141-140$ & 143726.7912 & $(8552)$ & 335.652 \\
$142-141$ & 144745.4299 & $(8736)$ & 340.446 \\
$143-142$ & 145764.0538 & $(8923)$ & 345.275 \\
$144-143$ & 146782.6627 & $(9112)$ & 350.137 \\
$145-144$ & 147801.2565 & $(9304)$ & 355.033 \\
$146-145$ & 148819.8352 & $(9499)$ & 359.963 \\
$147-146$ & 149838.3987 & $(9697)$ & 364.927 \\
$148-147$ & 150856.9467 & $(9897)$ & 369.925 \\
$149-148$ & 151875.4793 & $(10099)$ & 374.957 \\
$150-149$ & 152893.9963 & $(10305)$ & 380.023 \\
$151-150$ & 153912.4977 & $(10513)$ & 395.123 \\
$152-151$ & 154930.9832 & $(10724)$ & 395.257 \\
$153-152$ & 155949.4529 & $(10938)$ & 400.627 \\
$154-153$ & 156967.9065 & $(11155)$ & 405.863 \\
$155-154$ & 157986.3441 & $(11375)$ & \\
\hline
\end{tabular}

